\title{
Visualization of Nerve Fiber Orientations in the Human Optic Chiasm Using Photomicrographic Image Analysis
}

\author{
Neeranjali S. Jain, ${ }^{1,2}$ Swaranjali V. Jain, ${ }^{1,2}$ Xiaofei Wang, ${ }^{3}$ Andrew J. Neely, ${ }^{3}$ Murat Tahtali, ${ }^{3}$ \\ Sanjiv Jain, ${ }^{4,5}$ and Christian J. Lueck ${ }^{1,5}$ \\ ${ }^{1}$ Department of Neurology, The Canberra Hospital, Canberra, Australian Capital Territory, Australia \\ ${ }^{2}$ Faculty of Medicine, University of New South Wales, Kensington, Sydney, New South Wales, Australia \\ ${ }^{3}$ School of Engineering and Information Technology, University of New South Wales, Canberra, Australian Capital Territory, \\ Australia \\ ${ }^{4}$ Department of Anatomical Pathology, The Canberra Hospital, Canberra, Australian Capital Territory, Australia \\ ${ }^{5}$ Australian National University Medical School, Canberra, Australian Capital Territory, Australia
}

Correspondence: Neeranjali S. Jain, Department of Neurology, The Canberra Hospital, PO Box 11, Woden, ACT 2606, Australia; neeranjalijain@gmail.com.

Submitted: June 8, 2015

Accepted: September 10, 2015

Citation: Jain NS, Jain SV, Wang X, et al. Visualization of nerve fiber orientations in the human optic chiasm using photomicrographic image analysis. Invest Ophthalmol Vis Sci. 2015;56:6734-6739. DOI:10.1167/ iovs. $15-17443$
Purpose. Hemidecussation of fibers entering the optic chiasm from the optic nerves is well recognized. The reason why bitemporal hemianopia results from chiasmal compression has not been fully explained. There is still a paucity of data relating to the precise details of the routes that the nerve fibers take through the chiasm and, in particular, where and how nerve fibers cross each other. This information is important to understanding why crossing fibers are selectively damaged as a result of chiasmal compression.

Methods. An optic chiasm obtained at postmortem was fixed, stained, and sectioned to allow high-resolution photomicrographs to be taken. The photomicrographs were integrated to allow regions of interest across entire sections to be analyzed for fiber direction and crossing.

REsults. The results confirmed that fibers from the temporal retina pass directly backward in the lateral chiasm to the optic tract, whereas fibers from the nasal retina cross to the contralateral optic tract. Crossings take place in the paracentral regions of the chiasm rather than in the center of the chiasm (where the nerve fibers are traveling mostly in parallel). The paracentral crossing regions are distributed in a largely postero-superior to antero-inferior arrangement.

Concuusions. These findings clarify the precise locations and crossing angles of crossing nerve fibers in the chiasm. This information may help explain the clinical observation of junctional scotoma and will provide a much better basis for structural modeling of chiasmal compression which, in turn, will improve our understanding of how and why bitemporal hemianopia occurs.

Keywords: optic chiasm, bitemporal hemianopia, photomicrograph, image analysis, Wilbrand's knee
T he human optic chiasm contains approximately 2.4 million nerve fibers that run from the optic nerves anteriorly to the optic tracts posteriorly. ${ }^{1-4}$ Nerve fibers arising from the nasal half of each retina decussate in the chiasm to enter the contralateral optic tract, whereas fibers arising from each temporal hemi-retina pass directly backward into the ipsilateral optic tract. ${ }^{5-11}$

Compression of the optic chiasm by pituitary and other parasellar masses typically gives rise to bitemporal hemianopia. This phenomenon suggests that the decussating nasal fibers are selectively vulnerable. However, why they should be particularly vulnerable remains unclear. It has been suggested that decussating fibers are affected because they lie in the center of the chiasm, which is generally the point of maximal compression or stretching generated by the expanding mass. ${ }^{12,13}$ Another theory has suggested that these central fibers are selectively susceptible to ischemia due to regional variation in the anatomy of their blood supply. ${ }^{14}$ More recently, McIlwaine et al. ${ }^{15}$ postulated that decussating fibers are more vulnerable to chiasmal compression simply as a result of their geometry because they have a smaller contact area between nerves over which any compressive force is distributed.

Unfortunately, detailed anatomical information about the precise topographical arrangement of nerve fibers within the chiasm, something that is necessary to validate these theories, is limited. It is well accepted that temporal uncrossed fibers are located laterally in the chiasm, whereas decussating fibers are located centrally. Anatomical evidence of this segregation of crossed and uncrossed fibers has been provided by studies in old world primates ${ }^{5-8}$ as well as by two histologic studies in humans. ${ }^{16,17}$ However, the exact pathway that the decussating fibers take through the chiasm remains controversial. For example, a study of chiasmal anatomy conducted on a subject who had previously lost an eye described a loop of decussating fibers that detoured anteriorly into the contralateral optic nerve before continuing posteriorly into the contralateral optic tract. This loop was subsequently referred to as "Wilbrand's knee"18 and damage to this loop was accepted as the explanation for the well-recognized clinical phenomenon of junctional scotoma. Subsequent studies on primate optic chiasm and on 
humans undergoing surgery to divide the optic nerve at the optic nerve-chiasm junction have, however, suggested that "Wilbrand's knee" is likely to be an artifact of monocular enucleation. ${ }^{7,19}$

Recently, high-resolution diffusion tensor imaging (DTI) techniques have provided new insight into the complex microstructure of the optic chiasm: The central part of the chiasm appears to contain fibers traveling predominantly in parallel, whereas the paracentral areas contain complex fiber orientations. Unfortunately, these paracentral areas could not be characterized further due to the technical limitations of DTI. ${ }^{10,11}$

In summary, there is uncertainty about the precise pathways that the crossing fibers take through the chiasm. This limits the development of anatomically accurate models that can be used to help unravel the mechanism of bitemporal hemianopia. ${ }^{20,21}$

Histologic techniques coupled with computer-based image analysis now permit more accurate examination of nerve fiber architecture than was previously possible. Accordingly, this study was set up to examine nerve fiber distribution, along with the precise locations of nerve fiber crossings, using photomicrographic image analysis of histologic sections of the human optic chiasm.

\section{MeTHODS}

This study was conducted in accordance with the Declaration of Helsinki. The study was approved by the ACT Health Human Research Ethics Committee (ETH 1.14.020). Informed consent was obtained from the relative of the donor for the use of tissue.

\section{Tissue Preparation}

A human optic chiasm was obtained at autopsy from a 52-yearold male approximately 60 hours after death. Two needles were passed through the chiasm close to one optic nerve and the contralateral optic tract and then removed, the resulting holes subsequently served as fiduciary markers. After fixation in formalin (10\% in phosphate buffer, $\mathrm{pH} 7.2)$, the chiasm was stained with a modified silver stain to demarcate axons. Staining was conducted en bloc over 1 week so as to preserve tissue architecture. The tissue was subsequently embedded in wax, sectioned in the axial plane at $5-\mu \mathrm{m}$ intervals, mounted on glass slides, and dewaxed. Digitization of the sections of the chiasm was performed using an Axio Scan Slide Scanner (Carl Zeiss Microscopy, Jena, Germany) using a $\times 20$ objective. The photomicrographs were acquired at a resolution of $220 \mathrm{~nm}$ per pixel. The method is summarized in Figure 1.

Digitized images were aligned according to the fiduciary markers and then subdivided into smaller regions of interest (ROIs), each measuring $0.37 \times 0.28 \mathrm{~mm}$ using the software package ZEN (Carl Zeiss Microscopy, Jena, Germany).

\section{Image Analysis}

For each ROI containing nerve tissue, a histogram of nerve fiber orientation angles was derived using OrientationJ, an ImageJ plugin (http://imagej.nih.gov/ij/; provided in the public domain by the National Institutes of Health, Bethesda, MD, USA) based on structure tensors (Fig. 2).

To correct for artifactual peaks in the data caused by ROI edge detection, custom-written code and data-smoothing algorithms were implemented in MATLAB (Mathworks, Inc., Natick, MA, USA). After application of an empirical threshold, the angle representing the most prevalent fiber direction was represented graphically for each ROI. Regions of interest with a single peak could represent truly parallel fibers (Fig. 2) or fibers crossing in three dimensions ("broad bandwidth" pattern, Fig. 2). These were distinguished on the basis of the full width at half-maximum (FWHM) of the peak. Regions of interest with a FWHM less than the median value of that of the entire chiasm $\left(26^{\circ}\right)$ were designated "true parallel," whereas those with an FWHM greater than the median were designated "broad bandwidth." Regions of interest demonstrating more than one peak were regarded as "true crossings." The accuracy of the fiber populations detected was manually verified for each ROI in several sections of the chiasm.

\section{Image Presentation}

The dominant angles of each ROI were then superimposed on the original photomicrographic sections. Where more than one direction was above the threshold, the ROI was represented as a cross showing both fiber orientations. "True crossing" ROIs were darkly shaded and "broad bandwidth" ROIs were lightly shaded for easier visibility.

\section{Results}

Figure 3 shows the resulting photomicrographs of sections of the chiasm at various levels with overlaid fiber orientation and FWHM data for individual ROIs.

Fibers in the lateral part of the chiasm arriving from the optic nerves and departing in the ipsilateral optic tracts were arranged in parallel, running in an anterior-posterior direction. Fibers in the central portion of the chiasm were also arranged in parallel, but these fibers ran medio-laterally. Fibers were predominantly arranged in fascicles in the proximal optic nerves but this arrangement was not apparent within the chiasm or in the optic tracts.

The most complex fiber arrangements were noted in the paracentral areas of the chiasm at the junction of its medial and lateral parts. Regions of interest containing "crossing" fiber populations (i.e., those that contained two [or more] distinct fiber orientations) were predominantly located in these paracentral areas. The fiber populations tended to be arranged in interweaving bundles measuring approximately 30 to $50 \mu \mathrm{m}$ in diameter. Paracentral crossings were found more posteriorly in the superior parts of the chiasm and more anteriorly in the inferior parts. The inferior crossings extended anteriorly to the junction of the optic nerve and the chiasm (Figs. 3E, 3F).

The angles made between fiber populations as they crossed each other varied as a function of their location within the chiasm. Within the paracentral parts of the chiasm, most crossings involved nerve fibers oriented approximately perpendicular to each other. However, additional lower-angle antero-posterior crossings occurred in more lateral regions, whereas some lowerangle medio-lateral crossings were seen more medially.

No area of true crossing was detected by the algorithm in the middle portion of the chiasm. However, many crossings were occurring out of plane of the histologic sections, even in the central portion of the chiasm.

The boundary between areas of crossing in the paracentral part of the chiasm and the laterally confined parallel fibers coursing from the optic nerve to the optic tract was often not clearly demarcated. Regions of interest containing crossings were present toward the lateral edges of the chiasm in some sections.

\section{Discussion}

The relative paucity of detailed information on the precise fiber architecture of the human optic chiasm has hindered efforts to 


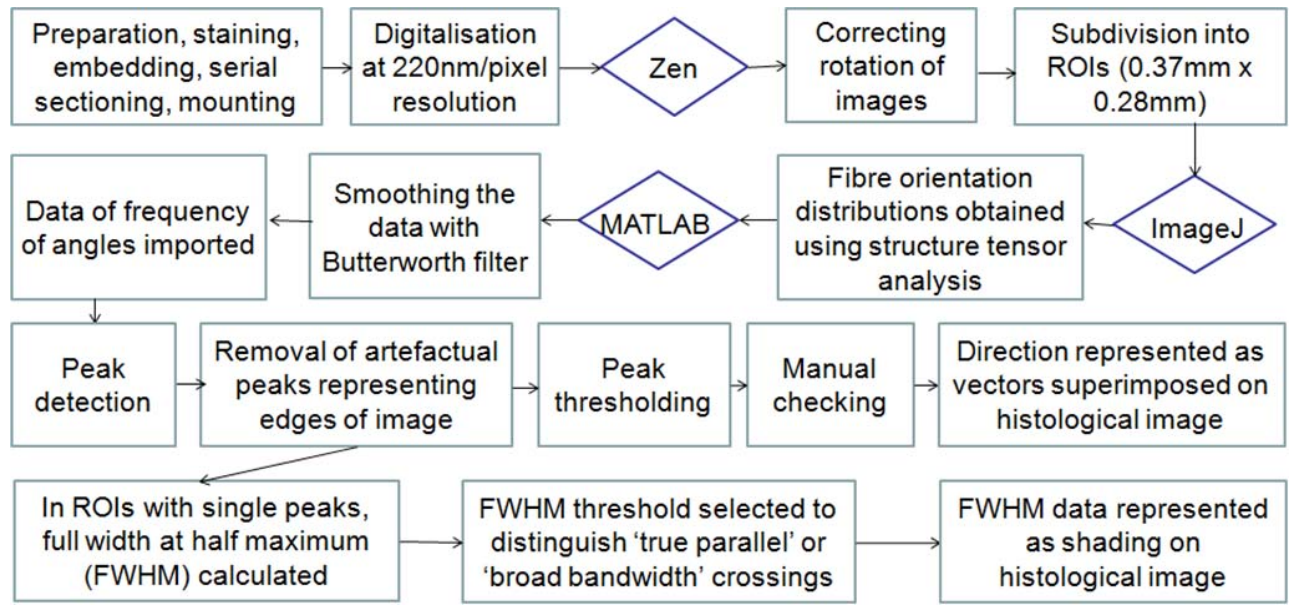

Figure 1. Summary of method. ImageJ, MATLAB, and ZEN are all proprietary software packages.
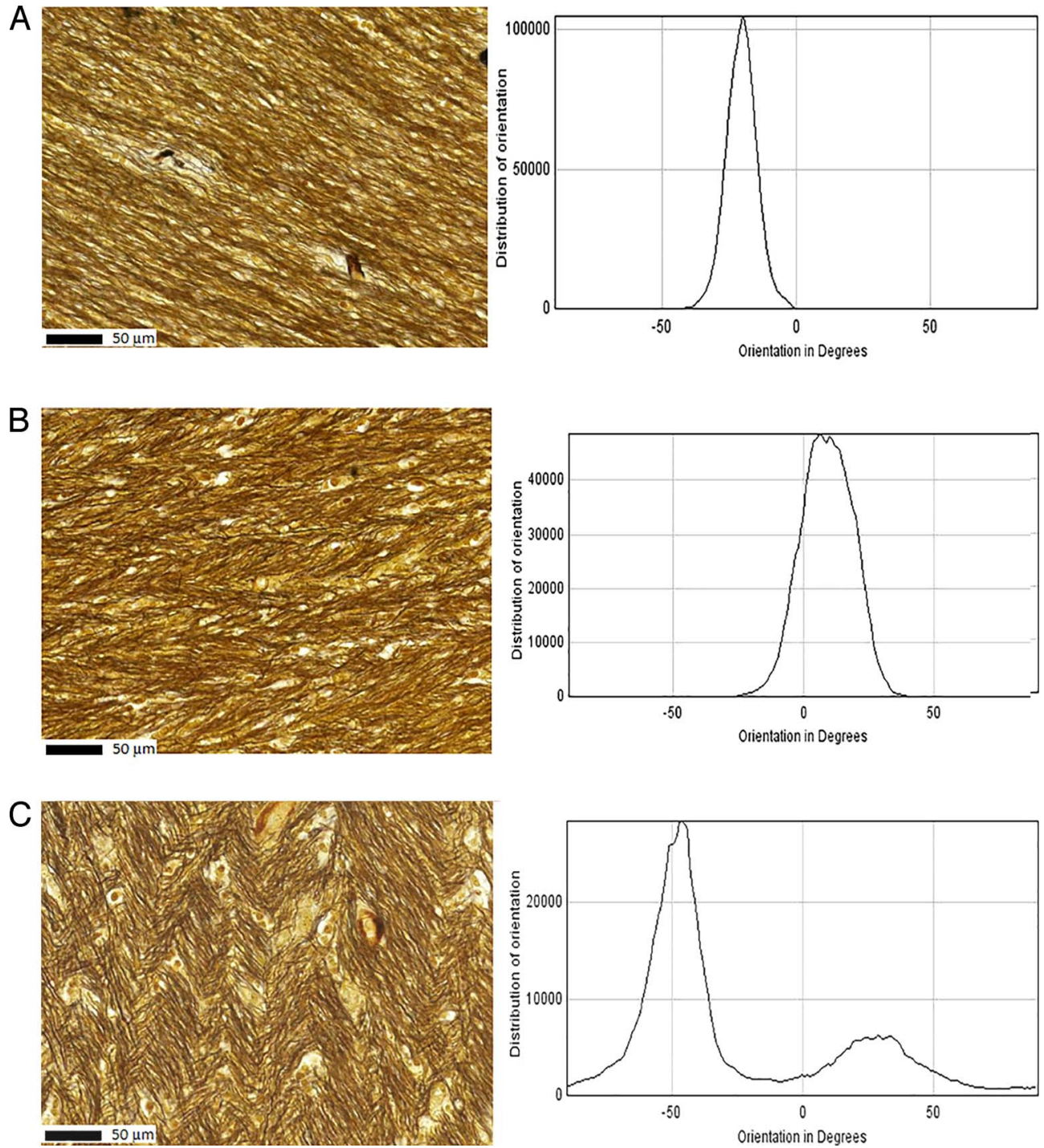

FigURE 2. Examples of ROIs with corresponding algorithm output. In (A), true parallel fibers are present, corresponding with a single peak with a narrow FWHM in the resulting histogram. In (B), fibers are crossing "out of plane," giving rise to a peak with a large FWHM. In (C), true crossing fibers are seen, giving rise to two separate peaks in the histogram; this result would be plotted as a cross on the images in Figure 3 . 
A

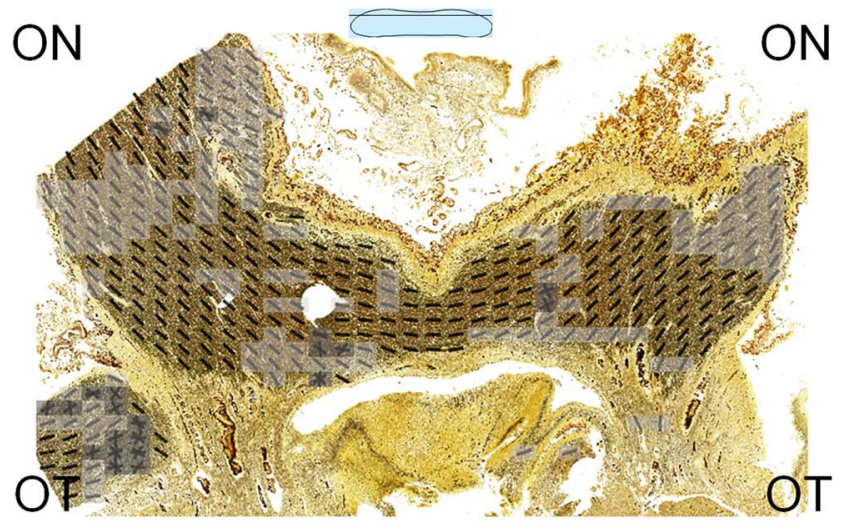

C

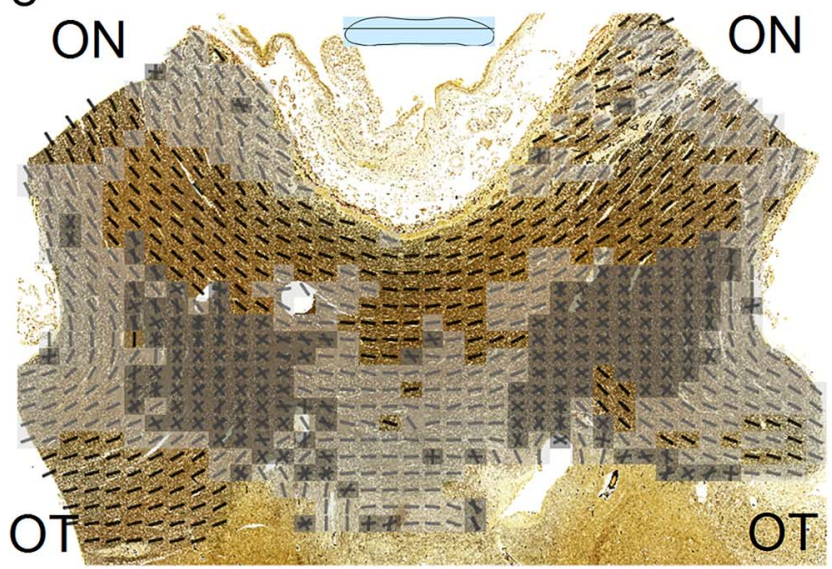

$\mathrm{E}$

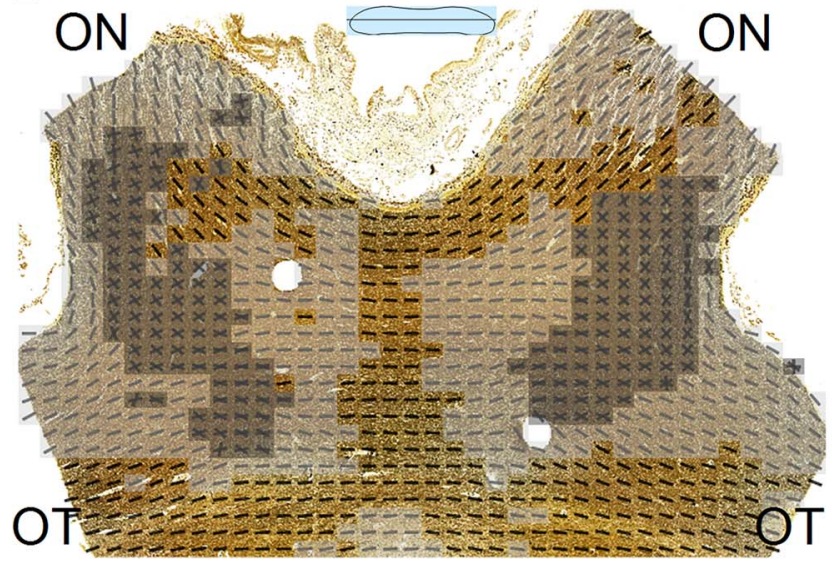

B

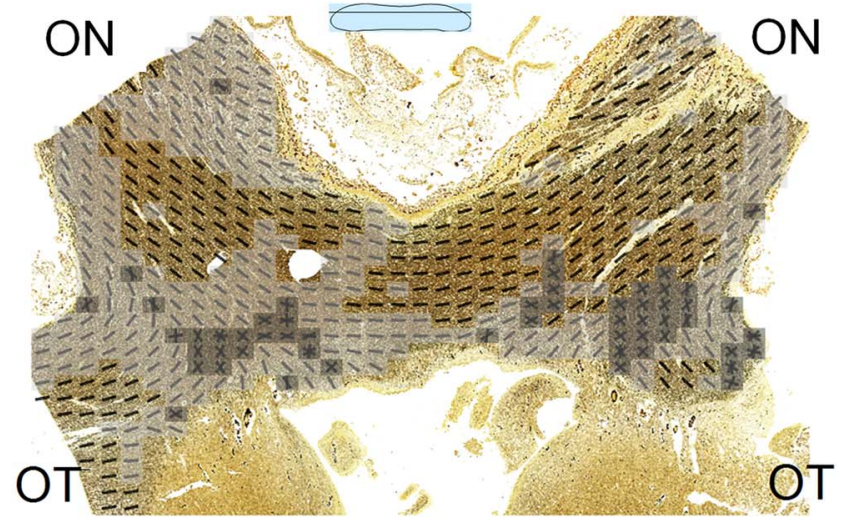

D

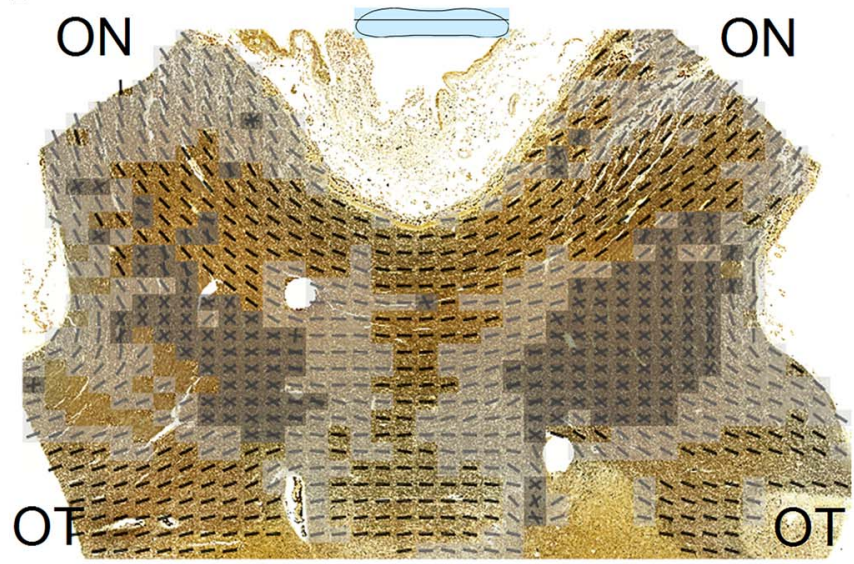

$\mathrm{F}$

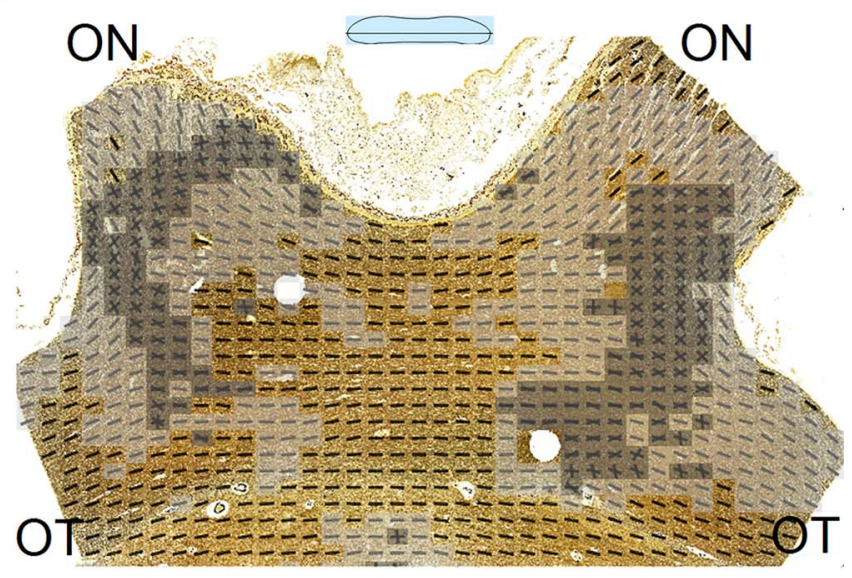

FiguRE 3. Photomicrographs of representative axial sections through optic chiasm arranged from superior (A) to inferior (F) showing dominant direction of axons for each ROI. ON, optic nerve; OT, optic tract. Regions of interest demonstrating two peaks are darkly shaded; ROIs demonstrating a FWHM bandwidth $>$ chiasmal median $\left(26^{\circ}\right)$ are lightly shaded. The inserts demonstrate the three-dimensional position of each section in the chiasm as a whole. Note that crossing fibers are located paracentrally and that there is a gradient: Areas of crossing are located more posteriorly in the higher sections (A-C), and more anteriorly in the lower sections $(\mathbf{E}, \mathbf{F})$.

explain the mechanism of the selective damage to nasal fibers in bitemporal hemianopia. Although it is well-accepted that nasal hemiretinal fibers decussate in the chiasm, the exact course of these fibers through the chiasm was unclear. This study has provided more detailed histologic analysis of the human optic chiasm with a view to elucidating nerve fiber arrangements and locations of fiber crossings. As expected, it confirmed that fibers arising from the temporal retina are located laterally in the chiasm, whereas nasal fibers decussate as they travel toward the contralateral optic tract. More specifically, the lateral and medial parts of the chiasm contained fibers that were oriented predominantly in parallel, whereas it was the paracentral parts of the chiasm that contained most of the fiber crossings. These crossings largely occurred at approximate right angles, although there was some variation depending on the precise location in the chiasm. 
The paracentral part of the chiasm has previously not been the subject of detailed examination in the literature, although recent studies have identified it as a region with a complex fiber microstructure. Roebroeck et al. ${ }^{10}$ used high-resolution DTI to examine an ex vivo human optic chiasm and found bilateral regions in the paracentral parts of the chiasm with substantial fiber curvature and crossing. The regions were described as diagonal bands running from posterosuperior to anteroinferior. These were postulated to represent nerve fiber crossings as nasal fibers diverged from the ipsilateral optic nerve and already decussated nasal fibers converged toward the contralateral optic tract. ${ }^{10}$ In a comparable high-resolution DTI study of an in vivo optic chiasm, Sarlls et al. ${ }^{11}$ found a "perimeter" around the central chiasm with low anisotropy, suggesting that multiple fiber orientations were present in this area. Neither DTI study was able to resolve the precise architecture of the nerve fibers in the paracentral chiasm due to the partial volume limitations of DTI.

Using histologic techniques to allow direct observation of fiber orientation, the current study was able to define the fiber architecture within the chiasm more clearly. The findings of this study are consistent with the findings of the DTI studies discussed above. ${ }^{10,11}$ Specifically, the ROIs with greatest fiber crossing were located in two zones running from the posterosuperior to anteroinferior aspects of the paracentral part of the chiasm based on the vector maps and corroboration by visual verification.

In addition to confirming the location of fiber crossings, this study provides further insight into the complex fiber organization in the paracentral regions. DTI studies have suggested that decussating fibers do not pass in a diagonally oriented path but curve sharply to adopt a medio-lateral direction through the central chiasm and then curve sharply once again to align with ipsilateral temporal fibers before entering the optic tracts. ${ }^{10,11}$ This arrangement correlates well with the pattern of fiber crossings seen in this study.

Most crossings in the paracentral regions involved nearperpendicular orientation of the nerve fibers. The medial edges of these regions contained crossings in which nerve fibers were arranged at lower angles in a medio-lateral direction, consistent with the mostly medio-lateral (i.e., transverse) orientation of fibers in the central part of the chiasm. Conversely, the crossings at the lateral edges of the paracentral regions occurred at lower angles in an anteroposterior direction. Previous work with finite element modeling has suggested that fibers that cross each other at angles of greater than $75^{\circ}$ are much more vulnerable to damage due to compression than those that cross at angles smaller than this; in particular, the model suggests that there is no additional vulnerability conferred by crossing angles lower than $25^{\circ} .22$ The anatomical findings of this study are therefore supportive of the hypothesis that it is because the decussating nerve fibers cross almost perpendicular to each other that they are selectively vulnerable to compression. ${ }^{15,20}$

The current study found high-angle crossings as far forward as the optic nerve-chiasm junction, but only in the inferior parts of the chiasm. Assuming the crossing fibers in this region are derived from the infero-nasal parts of the retina, damage at this location would be likely to give rise to a junctional scotoma (i.e., ipsilateral central scotoma and contralateral upper temporal defect). This may explain the clinical occurrence of junctional scotomas, even if Wilbrand's knee does not exist as originally described. ${ }^{7}$

As expected, longitudinal fibers in the lateral portions of the chiasm were observed to run directly backward from the ipsilateral optic nerve to the ipsilateral optic tract. It is well accepted that these lateral fibers represent temporal uncrossed fibers. ${ }^{5-8,16,17}$ In a histologic study of the human optic chiasm,
Neveu et al. ${ }^{16}$ demonstrated that these uncrossed fibers occupied the lateral $35 \%$ to $45 \%$ of the chiasm and were spatially distinct from the nasal decussating fibers. A surprising finding of the current study was that crossings sometimes occurred out toward the lateral edges of the chiasm. This may indicate that some nasal fibers course more laterally than has been previously realized.

A potential limitation of this study is that we present the results of only one specimen. In fact, another optic chiasm was studied in the process of developing the staining technique presented here, although the chiasm was not complete due to tissue lift-off from the slides. In this case, the fiber architecture was consistent. In particular, the central chiasm was seen to be dominated by fibers traveling in parallel with most of the crossings happening in the paracentral regions. Further detailed analyses with additional specimens will be conducted in the future to examine the extent of interindividual variation.

It is possible that tissue might have become altered in the time interval between death and processing. However, the deceased body was stored at $4^{\circ} \mathrm{C}$ before postmortem and no degenerative changes were detected in the photomicrographs.

Another limitation of the study is that the photomicrographs provided only a two-dimensional representation of fibers within the chiasm. The presence of "broad-bandwidth" ROIs suggested that many crossings were occurring "out of plane" (i.e., in three dimensions). Although the possible superior-inferior component of fiber crossing could not be examined in this study, dorso-ventral reorganization of decussating fibers has previously been described using other techniques. ${ }^{5,11,23}$ The vertical spatial arrangement of fibers could be visualized in a future study by examining sections from specimens taken in a sagittal plane. An important area for further study is to develop our technique to allow exploration of these three-dimensional relationships of fibers within the chiasm. Possible ways in which this might be achieved include the use of tracer injections or the development of more advanced algorithms that can link individual fiber endings between the two-dimensional histologic slices. Ultimately, a central question is why fibers crossing in the paracentral areas are particularly vulnerable to compression, giving rise to the characteristic bitemporal hemianopia. Further work with finite element models incorporating the more accurate anatomic data gathered from this study will be useful in attempting to provide an answer to this question.

\section{Conclusions}

Most high-angle nerve fiber crossings in the optic chiasm occur in the paracentral parts of the chiasm in a band running from posterosuperior to anteroinferior. This study has demonstrated that photomicrographic image analysis is an effective method of characterizing the microstructure of the human optic chiasm. Defining the geometric arrangement of nerve fibers is important, as it will permit more precise biomechanical modeling of chiasmal compression that may, in turn, provide greater understanding of why nerve conduction fails as a result of compression, and this may have implications for treatment. Further investigation with multiple optic chiasms and with three-dimensional reconstructions would be useful in building on the findings of this study.

\section{Acknowledgments}

The authors thank Mary Abbey and Jackie Simmonds from the Department of Anatomical Pathology at Canberra Hospital for preparing the histologic slices and Simon Kinder from Carl Zeiss Microscopy for digitizing the histologic slices. 
Supported by the Canberra Hospital Private Practice Fund and University of New South Wales Canberra Research Publication Fellowship (XW).

Disclosure: N.S. Jain, None; S.V. Jain, None; X. Wang, None; A.J. Neely, None; M. Tahtali, None; S. Jain, None; C.J. Lueck, None

\section{References}

1. Kupfer C, Chumbley L, Downer JC. Quantitative histology of optic nerve, optic tract and lateral geniculate nucleus of man. $J$ Anat. 1967;101:393-401.

2. Arey L, Bickel W. The number of nerve fibers in the human optic nerve. Anat Rec. 1935;61.

3. Potts AM, Hodges D, Shelman C, Fritz KJ, Levy NS, Mangnall Y. Morphology of the primate optic nerve. I. Method and total fiber count. Invest Ophthalmol Vis Sci. 1972;11:980-988.

4. Quigley HA, Addicks EM, Green WR. Optic nerve damage in human glaucoma. III. Quantitative correlation of nerve fiber loss and visual field defect in glaucoma, ischemic neuropathy, papilledema, and toxic neuropathy. Arch Ophthalmol. 1982; 100:135.

5. Hoyt WF, Luis O. Visual fiber anatomy in the infrageniculate pathway of the primate: uncrossed and crossed retinal quadrant fiber projections studied with nauta silver stain. Arch Ophthalmol. 1962;68:94-106.

6. Hoyt WF, Luis O. The primate chiasm: details of visual fiber organization studied by silver impregnation techniques. Arch Ophthalmol. 1963;70:69-85.

7. Horton JC. Wilbrand's knee of the primate optic chiasm is an artefact of monocular enucleation. Trans Am Ophthalmol Soc. 1997;95:579.

8. Naito J. Retinogeniculate projection fibers in the monkey optic chiasm: a demonstration of the fiber arrangement by means of wheat germ agglutinin conjugated to horseradish peroxidase. $J$ Comp Neurol. 1994;346:559-571.

9. Staempfli P, Rienmueller A, Reischauer C, Valavanis A, Boesiger $\mathrm{P}$, Kollias S. Reconstruction of the human visual system based on DTI fiber tracking. J Magn Reson Imaging. 2007;26:886893.

10. Roebroeck A, Galuske R, Formisano E, et al. High-resolution diffusion tensor imaging and tractography of the human optic chiasm at 9.4 T. Neuroimage. 2008;39:157-168.
11. Sarlls JE, Pierpaoli C. In vivo diffusion tensor imaging of the human optic chiasm at sub-millimeter resolution. Neuroimage. 2009; 47:1244-1251.

12. Hedges TR. Preservation of the upper nasal field in the chiasmal syndrome: an anatomic explanation. Trans Am Ophthalmol Soc. 1969;67:131-141.

13. Kosmorsky GS, Dupps WJ Jr, Drake RL. Nonuniform pressure generation in the optic chiasm may explain bitemporal hemianopsia. Ophthalmology. 2008;115:560-565.

14. Bergland R. The arterial supply of the human optic chiasm. $J$ Neurosurg. 1969;31:327-334.

15. Mcllwaine GG, Carrim ZI, Lueck CJ, Chrisp TM. A mechanical theory to account for bitemporal hemianopia from chiasmal compression. J Neuroophthalmol. 2005;25:40-43.

16. Neveu MM, Holder GE, Ragge NK, Sloper JJ, Collin JRO, Jeffery G. Early midline interactions are important in mouse optic chiasm formation but are not critical in man: a significant distinction between man and mouse. Eur J Neurosci. 2006;23: 3034-3042.

17. O'Connell J. The anatomy of the optic chiasma and heteronymous hemianopia. J Neurol Neurosurg Psychiatry. 1973;36:710-723.

18. Wilbrand HL, Saenger A. Die Neurologie des Auges. Ein Handbuch fur Nerven und Augenarzte. Wiesbaden, Germany: Bergman; 1904.

19. Lee JH, Tobias S, Kwon J-T, Sade B, Kosmorsky G. Wilbrand's knee: does it exist? Surg Neurol. 2006;66:11-17.

20. Wang X, Neely AJ, McIlwaine GG, Tahtali M, Lillicrap TP, Lueck CJ. Finite element modelling of optic chiasmal compression. $J$ Neuroophthalmol. 2014;34:324-330.

21. Wang $X$, Neely AJ, McIlwaine GG, Lueck CJ. Multi-scale analysis of optic chiasmal compression by finite element modelling. J Biomech. 2014;47:2292-2299.

22. Wang X, Neely A, Lueck C, Tahtali M, McIlwaine G, Lillicrap T. Parametric studies of optic chiasmal compression biomechanics using finite element modelling. In: Kotousov A, Das R, Wildy S, eds. Proceedings of the 7th Australasian Congress on Applied Mechanics. Barton, Australia: Engineers Australia; 2012;341-350.

23. Jeffery G. Architecture of the optic chiasm and the mechanisms that sculpt its development. Physiol Rev. 2001;81:13931414 . 